\title{
Prevalence of Anterior Knee Pain AMONG Young South African Basketball Players
}

\begin{abstract}
The aim of the article is to provide an indication of anterior knee pain prevalence among young South African basketball players. A retrospective questionnaire survey was conducted to capture information on injuries sustained over one season. The sample comprised 458 young basketball players in Cape Town and comprised 122 club players and 337 school basketball players. A total of 180 knee injuries were reported indicating a rate of 1 knee injury in 2.5 players. Anterior knee pain was the most commonly reported area of pain by the 458 basketball players $(49.1 \%, n=141)$. The most common combination of pain area was anteromedially $(18.2 \%, n=141)$. The results of our survey are in agreement with other studies indicating that patellofemoral pain is probably the most common knee presentation among young active individuals and arguably the most common condition seen at sports injury clinics.
\end{abstract}

KEY WORDS: KNEE , ANTERIOR KNEE PAIN, PREVALENCE OF ANTERIOR KNEE PAIN, BASKETBALL, INJURY MECHANISMS

\section{INTRODUCTION}

Knee and ankle injuries are by far the most common injury types sustained during basketball (Jones et al 2000, Micheli et al 1999). Basketball, though traditionally classified as a non-contact sport, is a dynamic sporting activity characterized by repetitive jumping, running, sharp changes in direction and sideways cutting manoeuvres. The loads and kinetic forces placed on the body during basketball may be related to the high prevalence of injuries, particularly to the lower limb.

Both traumatic and overuse type of knee conditions are reportedly common basketball injuries. Common conditions include ligamentous lesions, patellar tendonitis, muscle sprains, meniscal lesions, etc. (Messina et al 1999). Injuries resulting in anterior knee pain such as patellar tendonitis have been of particular interest to researchers due to the common nature of the presentation,

\section{CORRESPONDENCE TO:}

Quinette Louw

Department of Physiotherapy

University of the Western Cape

P.O. Box X17, Bellville 7535

Email: qlouw@uwc.ac.za

Table 1

\begin{tabular}{|l|l|l|l|l|l|}
\hline & Total (n) & $\mathbf{1 4}$ yrs & $\mathbf{1 5 y r s}$ & $\mathbf{1 6}$ yrs & $\mathbf{1 7 y r s}$ \\
\hline Boys & $68 \%(312)$ & $22,7 \%(104)$ & $16,4 \%(75)$ & $15.7(72)$ & $13.3 \%(61)$ \\
\hline Girls & $32 \% \quad(146)$ & $9.63 \%(44)$ & $13.79 \%(63)$ & $5.6 \%(26)$ & $2.8 \%(13)$ \\
\hline Mean age: 15.2 & SD 1.06 & & & & \\
\hline
\end{tabular}

poorly understood pathology and consequent difficulty in management of these conditions.

The aim of this study is to present data pertaining to knee injuries related to anterior knee pain among local, competitive young basketball players. Data on adolescent sports injuries are limited and often flawed by a number of factors such as recall bias, lack of exposure data, inappropriate sampling and recruitment procedures, etc. Difficulty in collecting data on overuse injuries has also been highlighted due to the difficulty in capturing the accurate nature and onset when injuries are self reported (Jones et al 2000) Consequently adolescent sports injuries are often misunderstood, misdiagnosed and the severity downplayed. Our data set based on a recently conducted retrospective survey provides an indication of the prevalence of anterior knee pain and selected associated factors in a South African sample and provides further insight into knee overuse injuries.

\section{STUDY DESIGN MEASUREMENT TOOL}

A retrospective questionnaire survey was conducted to capture information on injuries sustained. A previously validated questionnaire was adapted to collect data retrospectively over one season from South African basketball players and to provide detailed information regarding their knee injuries (Jones et al 2000). They could nominate all body parts injured during the past season and then reported details regarding a maximum of three most recent knee injuries sustained during the season.

\section{SAMPLE DESCRIPTION}

The sample comprised 458 young basketball players in Cape Town and comprised 122 club players and 337 school basketball players. Table 1 is a summary of the age and gender of the sample. Overall about $70 \%$ of the total population was 
captured. The response rate from the clubs was higher than the schools.

\section{RELIABILITY OF RESPONSES}

Reliability of the player responses was verified by surveying their parents. Eight percent of all parents were randomly selected as about 30 repeated measures were needed to determine reliability. Thirty-six parents were randomly selected by applying a customised Visual Basics program to the young players' data. The parents of these players were telephoned after completion of data collection to verify the responses of the young players as to whether an injury had been sustained during the season and to confirm the location of the injury.

\section{ETHICAL APPROVAL}

The Western Cape Basketball Association and The United School Sports Association of South Africa (USSASA) granted permission in writing to conduct the study. Ethical approval was obtained from The University of South Australia. A project information sheet was sent to all parents. Players who participated provided a signed consent form from their parents to their coach or the principal researcher.

\section{DATA ANALYSIS}

The questionnaire responses were entered into Excel and then analysed descriptively using SAS Version 8. Specific SAS programs were written to accommodate the multiple responses provided by the participants. Percentages, means and standard deviations were calculated to describe the data set. Probability calculations (odds ratios) were determined and significant risk was identified by 95\% confidence limits around odds ratios where neither $95 \%$ confidence limits encompass the value of 1 .

\section{RESULTS}

\section{General injury rate}

The general injury rate was high, with $186(40,6 \%)$ of the total sample reporting that they sustained at least one injury during the season indicating an injury rate of approximately one injury per two players. Players could report an unlimited number of injured body parts. A total of 304 injured body parts were reported.
Figure 1

\section{REPO RTED PAIN AREAS ( $\mathrm{N}=141)$}

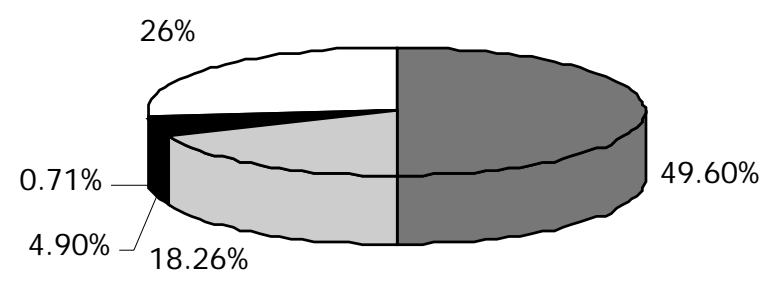

\section{Knee injury rate}

Ninety-seven players reported one or more knee injuries over the past season. The knee joint was the most commonly injured and the probability of sustaining a knee injury was also significantly more than sustaining an injury to another body part or sustaining no injury (Odds Ratio:1,31 :CI 1,15-1,5). Players could report a maximum of four knee injuries, but the questionnaire only made provision for details about three knee injuries. A total of 180 knee injuries were reported indicating a rate of 1 knee injury in 2.5 players.

The definition of injury incorporated both acute (injuries resulting from single macro-trauma) and overuse (injuries resulting from repetitive micro-trauma) injuries.

\section{Frequency of play}

Boys tended to play for longer periods per week compared with girls. The average number of hours per week played by boys was 7.2 (SD 4.2) and 5.9 (SD 3.6) by girls.

\section{Reported pain areas}

The most common symptom was pain $(23,87 \%)$ A total of 141 painful areas were reported based on the details provided about three knee injuries. The players could report the pain to be experienced as "anterior", "medially', "posterior", "laterally" or any combination of the these pain areas. The players most commonly reported anterior knee pain (Figure 1). The most common combina- tion of pain area was experienced anteromedially $(18.2 \%, \mathrm{n}=141)$. Only eight $(4.9 \% ; n=141)$ injuries resulted in anterolateral pain. Therefore $73 \%$ $(n=141)$ of all painful areas reported by these active young basketball players involved the anterior aspect of the knee joint.

\section{Anterior knee pain related to gender and age}

A total number of $71(73 \%)$ of the 97 knee injured players reported anterior knee pain. The 14-15 year old players $(16 \% ; n=286)$ reported relatively more anterior knee pain than the 16 - 17 year old players $(13 \% ; n=172)$.

About $16 \%$ of the boys $(n=312)$ and $13 \%$ of the girls $(n=146)$ reported anterior knee pain. However the odds that anterior knee pain is more common in boys than girls was not significant (OR 1.3:95\%CI 0.75-2.35).

\section{Injury mechanisms resulting in anterior knee pain}

Players could nominate multiple injury mechanisms and 85 injury mechanisms were reported to result in anterior knee pain as a symptom. Forty percent $(n=85)$ of these injuries were as a result of landing badly from a jump. Thereafter, the second most common mechanisms were "overuse pain" not preceded by a traumatic injury $(10,5 \%, n=85)$, followed by falling.

\section{Severity of anterior knee pain}

An indication of severity was obtained by the ability of the player to continue 
playing. About $20 \% \quad(n=141)$ of the injuries that involved anterior knee pain caused the players to stop playing. Eighteen injuries presenting with anterior knee pain caused players to stop for about one week, 9 injuries resulted in loss of play for more than a week and only 4 injuries resulted in loss of play for more than one month.

\section{Management}

Most knee injuries were not treated at all $(63.46 \%)$. Only $24 \%(n=141)$ of the injuries leading to anterior knee pain were treated.

Severe knee injuries were treated more often than minor injuries. About $25 \%$ of the knee injuries resulting in loss of play were treated and only $20 \%$ of injuries that did not result in loss of play were treated.

The initial knee injuries were less well managed. This is evidenced by $35 \%(n=143)$ of the initial injuries being treated, $36 \%$ of the second injury sustained were managed and $50 \%$ of the third injury were treated.

Most injuries were conservatively managed with only medication. A small number of injuries were managed by physiotherapy $(7.6 \%)$. Notably conservative management such as 'medication and rest' $(5,6 \% n=143)$ was the most common form of treatment and was the most frequently reported combination of treatment methods.

\section{DISCUSSION}

The general high injury rate $(40.6 \%)$ challenges the classification of basketball as a non-contact sport perceived to be safer than contact sports. Vulnerability to injury may be enhanced by the apparently more aggressive level of play combined with underdeveloped skill often displayed by adolescents. Injury is a common reason for young people to cease participation in physical activity (Du Randt et al 1991). Health professionals such as physiotherapists should thus be concerned considering the health related benefits of physical activity.

The high rate of knee injuries is in agreement with previous studies (Jones et al 2000, Louw et al 2002). However the knee injury rate of $30 \%$ is higher than reported in previous studies that report a knee injury rate of about $20 \%$ (Arendt and Dick 1995). This may be related to the injury definition employed in this study. Most previous studies only collected information on traumatic injuries resulting in loss of play whereas this study included both traumatic and atraumatic injuries. Omitting overuse injuries in basketball injury surveillance may lead to an underestimate of injuries due to the common nature of overuse injuries such as patellar tendonitis among basketball players (Hicky et al 1997).

The high prevalence of anterior knee pain in this sample could possible serve as an indication of the high rate of overuse injuries in basketball. About $73 \%$ of pain areas involved the anterior aspect of the knee. The most common combination of pain area nominated was anteromedial knee pain and this is in agreement with previous studies (Powers 1998). Many overuse conditions such as patellar tendonitis, maltracking of the patella, etc. present clinically with anterior knee pain. However this assumption must be viewed with caution as $40 \%$ of injuries presenting with anterior knee pain were caused from landing badly from a jump and only $10 \%$ of the injuries were not preceded by a specific traumatic event. This highlights the difficulty in collecting data on overuse injuries and well validated methods should be developed to ensure reliable information of overuse injuries.

Landing is a complex task and requires significant skill and coordination. Adolescents may have difficulty in mastering this technique due to impaired coordination and inflexibility (Dalton 1992). In this sample younger players reported a higher prevalence of anterior knee pain than older players. Poor balance results in landing with the knee in either a large varus or valgus moment placing abnormal strain on the knee and rendering young players vulnerable to injury (Hewett et al 1996).

About $20 \%(n=141)$ of the injuries that involved anterior knee pain caused players to stop playing and it is thus startling that most of these injuries were not treated. The growth rate of basketball since 1992 in South Africa is mostly attributed to the increase in players from relatively poorer socio-economic back- grounds. More than $80 \%$ of the registered competitive players in the Western Cape are from communities previously considered as disadvantaged. Parents may not be able to afford or identify appropriate management and Basketball South Africa does not have medical support structures for injured players.

A very small number of players consulted a physiotherapist. Most of the players who had treatment used medication. Education of all participants such as coaches, parents, players, teachers etc. is needed to encourage dynamic rehabilitation of injuries as opposed to more traditional medical management approaches. The small number of players who consulted a physiotherapist may also be a reflection of inadequate promotional programs conducted by physiotherapists. Physiotherapists adopt a proactive approach to focus on preventative aspects as well as rehabilitative programs. The discrepancy between the number of players who received physiotherapy and medical treatment may indicate that interdisciplinary health management should be re-emphasised. Medical practitioners are not always aware of the type of conditions treated by physiotherapists since this does not form part of their formal training (Lee and Sheppard 1998). Consequently appropriate clients are not referred for further management and players may be at risk of injury recurrence due to inadequate preparation to ensure safe return to sport.

\section{CONCLUSION}

The prevalence of anterior knee pain among young South African Basketball players is high. Epidemiological information provides important information that aids in understanding the scope and aetiology of the problem. More robust studies are however required to provide reliable epidemiological information on overuse knee injuries that could lead to effective preventative strategies.

\section{ACKNOWLEDGEMENTS}

The Medical Research Council granted financial support for the survey

Denric Blaauw, Laura Radcloff and Wilfred Allen for assisting with data collection 


\section{REFERENCES}

Arendt E and Dick R (1995): Knee injury patterns among men and women collegiate basketball and soccer. The American Journal of Sports Medicine; 23(6): 694-701

Dalton S E (1992): Overuse injuries in adolescent athletes. Sports Medicine 13: 58-70

Du Randt R, Pendergrast $\mathrm{R}$ and Donner $\mathrm{J}$ (1991): Adolescents' attrition from school sponsored sports. Sports Medicine 145: 11191123

Hewett T, Stroupe A, Nance T and Noyes F (1996): Plyometric training in Female athletes: The American Journal of Sports Medicine 24(5):765-773

Hicky G, Fricker P and McDonald W (1997): Injuries to young elite basketball players over a six-year period. Clinical Journal of Sports Medicine: 7: 252 - 256
Jones D, Louw Q, Grimmer K (2000): Recreational and sporting injury to the adolescent knee and ankle: Prevalence and causes. Australian Journal of Physiotherapy; 46: 3: 179-188

Lee K and Sheppard L (1998): An investigation into medical students; knowledge and perception of physiotherapy services. Australian Journal of Physiotherapy 44 (4): 239-45

Louw Q, Grimmer K and Vaughan K (2002): Knee injury patterns among young basketball players in Cape Town. South African Sports Medicine Journal (in press: January 2002 edition)

Messina D, Farney W and De Lee J (1999): The incidence of injury in Texas high school basketball. The American Journal of Sports Medicine: 27(3):294-299
Micheli L, Metzl J, Di Canzio D (1999): Anterior reconstructive surgery in adolescent soccer and basketball players. Clinical Journal of Sports Medicine:9(3): 138-41

Powers C (1998): Rehabilitation of the patellofemoral joint disorders: A critical review. Journal of Orthopaedic and Sports Physical Therapy 28(5):345-354

\section{JustPhysio introducing Anton Shaw with
10 years experience successfully recruiting locum physiotherapists to the UK \\ JustPhysio with extensive work opportunities throughout the UK, excellent rates of pay, conditions and full benefits package}

\section{Just call Anton Shaw on freephone 0800990946}

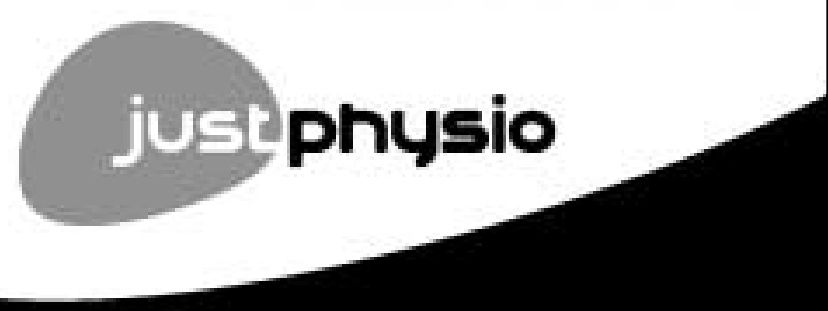

Lawtord House, Albert Place, Finchley, London, N3 1QA, UK Tel: +44 (0) $2083713535 \mathrm{Fax}+44$ (0) 2083713530

email: anton.shaw wWw.justphysio.co.uk

\section{Develop your Career in the UK}

Immediate vacancies throughout the UK for Physiotherapists...

*Visa fee re-imbursement

- Immigration advice *

Meet and greet service *

Accommodation assistance *

Bank account •

Excellent pay •

Free financial advice *

Personal development fund •

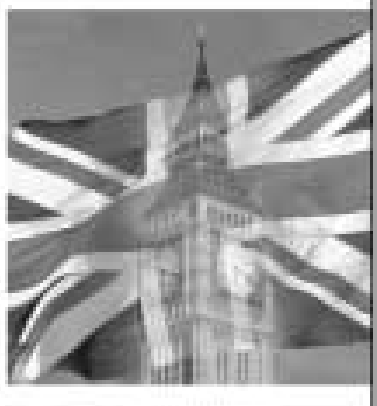

Cape Town:

Pretoria

(021) $6805146 \quad$ (012) 3624523

THERAPY sardi our plotse

www.therapy-workline co.uk

E southaficsetherapy workine co,uk 